\title{
Recurrent pregnancy loss is associated with unfavourable composition of endometrial and vaginal microbiota.
}

Pirkko Peuranpää ( $\nabla$ pirkko-liisa.peuranpaa@helsinki.fi )

The Department of Obstetrics and Gynecology, University of Helsinki and Hyvinkää Hospital, Hyvinkää, Finland https://orcid.org/0000-0002-6841-0757

\section{Research Article}

Keywords: Recurrent pregnancy loss, recurrent miscarriage, microbiota, endometrium, vagina

Posted Date: February 18th, 2022

DOI: https://doi.org/10.21203/rs.3.rs-1167008/v2

License: (c) (1) This work is licensed under a Creative Commons Attribution 4.0 International License.

Read Full License 


\section{Abstract}

Research Question Does endometrial or vaginal microbiota composition associate with recurrent pregnancy loss (RPL)?

Design Endometrial and vaginal samples were collected from 47 women with two or more consecutive pregnancy losses and 39 healthy control women without a history of pregnancy loss between March 2018 and December 2020 in Helsinki University Hospital, Helsinki, Finland. The endometrial and vaginal microbiota compositions, analysed using 16S rRNA gene amplicon sequencing, were compared between RPL and control women, and between individual vaginal and endometrial samples. The mycobiota composition was analysed using internal transcribed spacer 1 amplicon sequencing for a descriptive summary. The models were adjusted for $\mathrm{BMI}$, age, and parity. False discovery rate -corrected $P$-values ( $q$ values) were used to define nominal statistical significance at $q<0.05$.

Results Lactobacillus crispatus was less abundant in the RPL women's endometrial samples compared to controls (mean relative abundance $17.2 \%$ vs. $45.6 \%, q=0.04$ ). Gardnerella vaginalis was more abundant in RPL women than in controls in both endometrial $(12.4 \%$ vs. $5.8 \%, q<0.001)$ and vaginal samples $(8.7 \%$ vs. $5.7 \%, q<0.01)$. The individual vaginal and endometrial microbial compositions correlated strongly $(R=0.85, P<0.001)$. Fungi were detected in $22 \%$ of the endometrial and $36 \%$ of the vaginal samples.

Conclusions Unfavourable reproductive tract microbiota is associated with RPL and may represent a novel risk factor for pregnancy losses.

\section{Key Message}

Lactobacillus crispatus was less and Gardnerella vaginalis more abundant in endometrial samples of 47 women with recurrent pregnancy loss, compared to 39 healthy control women. Unfavourable endometrial microbiota may be a novel risk factor for RPL, a condition that currently often remains unexplained in standard examinations.

\section{Introduction}

Recurrent pregnancy loss (RPL) is one of the most challenging issues in reproductive medicine because its causes are often unknown, and effective treatment is rarely available. European Society of Human Reproduction and Embryology (ESHRE) defines RPL as the spontaneous loss of two or more pregnancies (Bender Atik et al., 2018), and it affects 1-3\% of couples trying to have a child. Recognised causes for RPL are chromosomal abnormalities, uterine malformations, antiphospholipid syndrome, and endocrinological disorders (Rai and Regan, 2006), while over half of the RPLs still remain unexplained. 
Several studies have suggested an infectious aetiology behind miscarriages (Giakoumelou et al., 2016; McQueen et al., 2015). Chronic endometritis has been associated with RPL (McQueen et al., 2021) and bacterial vaginosis (BV) has been linked to the risk of miscarriage (Haahr et al., 2019; Ralph et al., 1999). Recent studies using high-throughput DNA sequencing techniques have shown that Lactobacillus spp. dominate the vaginal bacterial composition in healthy early pregnancy (Freitas et al., 2017; Maclntyre et al., 2015), while the reduced prevalence of lactobacilli has been associated with pregnancy loss (Al-Memar et al., 2020). Mycobiota, consisting of various species of fungi, is another important element of the vaginal ecosystem (Bradford and Ravel, 2017). However, there are no studies examining mycobiota in relation to reproductive outcomes.

The uterine cavity has long been thought to be sterile, but recent studies have reported that endometrium may have a distinct microbiome (Chen et al., 2017; Koedooder et al., 2019). Like in the vagina, the dominant species in the endometrium are usually lactobacilli (Chen et al., 2017, Oberle et al., 2021), and alteration of this composition may affect reproductive outcomes. In patients treated with in vitro fertilization (IVF), non-Lactobacillus dominated endometrial microbiota has been associated with lower implantation rates, clinical pregnancy rates, and live birth rates compared to Lactobacillus-dominated microbiota (Moreno et al., 2016). The impact of endometrial microbiota on RPL has not been investigated before.

The goal of our study was to explore the composition of microbiota and mycobiota in endometrial and vaginal samples in women with RPL and compare the results to healthy women without a history of miscarriages. We also investigated whether the composition of vaginal microbiota reflects the composition of endometrial microbiota.

\section{Material And Methods}

\section{Study population}

TOIVE is a prospective cohort study of the immunological and microbiological causes of RPL, conducted at the University of Helsinki and Helsinki University Hospital (HUS), Finland. Between March 2018 and June 2020, we recruited 51 women referred to the Reproductive Medicine Unit and the Department of Obstetrics and Gynaecology of Hyvinkää Hospital due to RPL (Figure 1). Women were eligible if they had a history of three or more consecutive clinical first-trimester pregnancy losses or two losses, with at least one of them in the second trimester. We defined clinical pregnancy loss as a spontaneous loss of an intrauterine pregnancy confirmed by an ultrasound, or positive urine or serum human chorionic gonadotrophin and over six weeks of amenorrhea. 
As controls, we recruited 40 women investigated for male factor infertility between June 2018 and December 2020. They represented healthy Finnish women since they had no history of pregnancy losses, endometriosis, anovulation, or fallopian tube defects. Participants were excluded if they were $<18$ or $\geq 40$ years of age, had hepatitis or HIV infection, or had an irregular menstrual period ( $<21$ or $>42$ days).

Power calculations were not applicable to our associative and novel setting since earlier studies on microbiota composition and RPL are lacking. The ethics committee of HUS approved the study (No. HUS/3635/2017).

\section{Collection of clinical data}

All participants gave their written informed consent. Upon enrolment, we took a detailed medical and reproductive history, and participants received a questionnaire related to previous infections, the use of anti- or probiotics, sexual behaviour, and educational background. The basic examinations for RPL included transvaginal 2D-ultrasound, complete blood count, phospholipid antibodies, karyotyping (of both partners), thyroid function tests, thyroid peroxidase antibodies, fasting glucose, and glycosylated haemoglobin. Screening for thrombophilia, celiac disease, hyperprolactinemia, or uterine anomalies with 3D-ultrasound or hysteroscopy were performed when necessary. Women were not routinely tested for sexually transmitted diseases nor chronic endometritis.

\section{Endometrial and vaginal sampling}

We collected vaginal and endometrial samples in the mid-luteal phase, 6-8 days after a positive ovulation test (Clearblue Digital ${ }^{\circledR}$, Swiss Precision Diagnostics $\mathrm{GmbH}$, Switzerland). Couples were advised to use a condom or abstain from sexual intercourse during the menstrual cycle in which the samples were taken for contraception and to avoid the effects of seminal fluid on the microbiota. Vaginal samples were collected in a speculum examination from the right and left fornix with sterile flocked swabs (FLOQSwabs, Copan spa ${ }^{\circledR}$, Italy) and severed to $1.5 \mathrm{~mL}$ Eppendorf tubes. We used non-sterile examination gloves and a white coat during sampling, but not a mask or hair cover. Lubricants were not used. Endometrial samples were collected to $1.5 \mathrm{~mL}$ Eppendorf tubes with endometrial biopsy curette (Pipelle ${ }^{\circledR}$, Prodimed, France) without contact to vaginal walls. Eppendorf tubes were frozen at $-20^{\circ} \mathrm{C}$ immediately after sampling and moved to $-80^{\circ} \mathrm{C}$ within two weeks.

\section{DNA extraction}


Microbial DNA was extracted from the vaginal samples using a beat beating method as previously described (Virtanen et al., 2019). One-sixth of the endometrium biopsies (average weight $33 \mathrm{mg}$; SD 22) was used for DNA extraction with negative controls using the same method as for the vaginal samples. DNA was quantified using Quanti-iT Pico Green dsDNA Assay (Invitrogen ${ }^{\circledR}$, San Diego, CA, USA). The DNA yields for both sample types were comparable; mean 78.8 (SD 60) $\mathrm{ng} / \mu \mathrm{l}$ in vaginal and 43.8 (SD 30) $\mathrm{ng} / \mu \mathrm{l}$ in endometrial samples. The negative controls (no input sample for DNA extraction) did not contain detectable amounts of DNA.

\section{S rRNA gene and Internal transcribed spacer (ITS) amplicon sequencing}

Illumina MiSeq paired-end sequencing of PCR-amplicons from the hypervariable V3-V4 regions of the bacterial 16S rRNA gene (primers 341F/785R) and fungal ITS-1 region (primers ITS1F and ITS2) was prepared and performed as explained in detail elsewhere (Virtanen et al., 2021). Briefly, bacterial and fungal amplicons were prepared separately, combined for indexing in a 1:1 ratio, and an equimolar pool was sequenced using $2 \times 300$ bp reads and a MiSeq v3 reagent kit at the Biomedicum Functional Genomics Unit (FuGU), Helsinki, Finland.

\section{Sequence processing and analysing}

The primary step of the analysis was to split the combined 16S rRNA gene and ITS sequence fastq-files into separate datasets. This was done by first removing ambiguous $(\mathrm{N})$ bases from the reads, followed by primer-based separation using cutadapt v3.5, removing the primers in the process. From this step onwards, all pre-processing was carried out individually on both datasets. The bacterial 16S rRNA gene dataset was processed using dada2 v1.20, following the pipeline tutorial v1.16, while the ITS pipeline tutorial v1.8 was used for processing the ITS sequence dataset.

The dada2 tutorials were only followed until ASV table construction and removal of chimeras, after which the taxminer package was used to assign taxonomic annotations (Saqib, 2021). This is a BLAST-based annotation tool combined with text-mining-based filtration to assign the most likely annotations. A detailed description of this approach has been communicated elsewhere (Oksanen et al., 2020).

\section{Statistical analysis}

Statistical analyses were performed using IBM SPSS Statistics, version 25 (IBM Corporation, Armonk, NY, USA) software for univariate data. Two-sample t-test and Mann-Whitney $U$ test compared the continuous background variables, Pearson chi-square, and Fisher's exact tests compared the categorical variables between RPL women and controls. Parity was categorized as nulliparous and parous ( $\geq 1$ delivery). 
The primary outcomes were mean relative abundances of bacteria and fungi in the endometrial and vaginal samples. Associations between background variables and the microbiota were analysed with permutational analysis of variance (PERMANOVA) for the overall microbiota variation using the adonis2 function with 99999 permutations from the vegan package (18). Potential confounders to adjust models for differential abundance analysis downstream were identified based on the following criteria: 1) factors previously identified to influence microbiota and RPL and 2) confounders with statistical significance, determined by PERMANOVA analysis, ordination plots, and the GroupTest function from the package mare (Korpela, 2016). GroupTest performs differential abundance analysis for taxa within user-defined groups, in this case each background variable. The function finds the most suitable model for individual taxa and uses read counts as an offset within the model formula to account for sequencing depth. Lastly, cases and controls were used as the grouping variable in GroupTest to determine the significantly different taxa in endometrial and vaginal samples between RPL and control women. Based on the criteria mentioned above, body mass index (BMI), parity, and age were selected as confounders. The $P$-values obtained were adjusted for multiple testing by False Discovery Rate (FDR)-correction and reported as $q$ values. The relationship between individual vaginal and endometrial microbiota was investigated using Pearson correlation coefficient.

\section{Results}

We sampled 47 women with RPL and 39 control women to study the composition of the endometrial and vaginal microbiota and mycobiota. In one RPL woman, only the vaginal sample was obtained. RPL women had a history of three (range 2-5) consecutive miscarriages, of which $13(15.3 \%)$ had occurred during the second trimester of pregnancy. Nine women had a likely explanation for their miscarriages: five were diagnosed with congenital uterine malformation, three with acquired thrombophilia, and one with antiphospholipid syndrome and chromosomal translocation.

The RPL women were older (mean 33.2 [22-39] vs. 32.1 [26-38] years, $P=0.04$ ), had a higher BMI (mean $25.4[19.5-39.4]$ vs. $23.3[19.4-33.1] \mathrm{kg} / \mathrm{m} 2, P=0.02)$, and were parous more often than the controls $(50.0 \%$ vs. $15.4 \%, P<0.001)$ (Table S1). The RPL women had more often self-reported recurrent $(\geq 3)$ candida vaginitis, $\mathrm{BV}$, and regular oral or vaginal probiotics use than the controls.

\section{S rRNA gene sequencing results}

After taxonomic annotations and quality filtration (> 500 reads), 74\% (34/46) of the RPL and 77\% (30/39) of control women's endometrial samples remained for bacterial analysis with an average read count of 4 500 (535-20 829), and 4 500 (569-11 540), respectively. Similarly, 98\% of the vaginal RPL samples 
(46/47) and 100\% of controls (39/39) remained, with an average read count of 23600 (3 728-47 923) and 24300 (1 322-55 011).

PERMANOVA analysis showed a difference in the overall endometrial microbiota composition between the RPL and control women (R2 $=0.050, P=0.01$, Figure S1, Table S2), but the vaginal bacterial compositions were similar between the groups (Figure S2, Table S2). BMI was a strong clinical explanatory factor for the endometrial microbiota $(\mathrm{R} 2=0.057, P=0.005)$, especially among RPL women (R2 $=0.09, P=0.005)$. Current vaginitis symptoms, use of vitamin $\mathrm{D}$ and folic acid, microbiota diversity, and read count explained the variability of the vaginal microbiota in the whole study population, and among the RPL women also BMI, age, and probiotics. Parity, gravidity, or curettages had no impact on the endometrial or vaginal microbiota variation.

In the endometrial samples, 37 bacterial species were identified (Figure 2A). L. crispatus was significantly less and $L$. jensenii more abundant in the RPL women compared to controls (mean relative abundance of L. crispatus $17.2 \%$ vs. $45.6 \%$, respectively, $q=0.04$, L. jensenii $5.6 \%$ vs. $3.6 \%, q<0.01$ ) (Figure 3, Table S3). L. iners was the most dominant endometrial bacterium in the RPL women (mean relative abundance $32.2 \%$ vs. $20.0 \%, q>0.05$ ). Gardnerella vaginalis was more abundant in the RPL women compared to controls $(12.4 \%$ vs. $5.8 \%, q<0.001)$. None of the intestinal bacteria, such as Escherichia coli, Blautia spp., and Faecalibacteria spp., nor the uncultured bacteria (bacteria that lack culture-based genomic and physiological characterization) were significantly differentially abundant in the RPL women compared to the controls.

In the vaginal samples (Figure 2B), the RPL women had more G. vaginalis (mean relative abundance $8.7 \%$ in RPL women vs. $5.7 \%$ in controls, $q<0.01$ ) and less $G$. Leopoldii (1.0\% vs. $3.2 \%$, respectively, $q<0.001$ ), whereas $L$. crispatus was the most abundant bacterium (35.1\% vs. $47.5 \%, q>0.05$ ) (Figure 3, Table S3). In the pooled endometrial and vaginal samples, $L$. crispatus was less and $G$. vaginalis more abundant in RPL women than in controls (L. crispatus $27.5 \%$ vs. $46.6 \%, q=0.04$; G. vaginalis $10.2 \%$ vs. $5.7 \%, q<$ $0.001)$.

There were three blank samples within this pipeline that were analysed with the same pre-processing and filtration criteria. Only two out of these three samples had a considerable number of reads, which totalled to $\sim 8500$ (Figure S3). These annotated to Streptococcus oralis ( 4000), Fannyhessia vaginae ( 600), Prevotella amnii ( 1700), and Sneathia vaginalis ( 1300). S. oralis, a potential oral contaminant, was not detected in any other samples. F. vaginae, $P$. amnii, and $S$. vaginae are known vaginal microbes, and their presence in the blank sample is most likely due to contamination from the primary study samples. Due to 
these reasons, we did not subtract these reads from the sample data. Furthermore, none of these bacteria were significant in our downstream analysis, and therefore not crucial for the main study comparisons.

\section{ITS sequencing results}

After taxonomic annotations and quality filtration, 24\% (11/46) of RPL and 21\% (8/39) of control women's endometrial samples remained for the fungal analysis, with an average read count of $\sim 14500$ (1031-81 809) and 12 000 (1383-23 999) respectively. Similarly, 36\% (17/47) of the RPL and 36\% $(14 / 39)$ of control women's vaginal samples remained after filtration, with an average read count of 6000 (742-22 500) and 13 000 (542-30 685). Since fungi could only be detected in a small fraction of the samples, they may not sufficiently represent the entire study cohort. We therefore refrain from statistical analysis and present a descriptive overview of the taxonomic profiles.

The most prevalent genus was Candida, which was detected in 19/39 samples (Figure S4). C. albicans was not detected in RPL women's endometrium, although it was the most abundant taxa in their vaginal samples (mean relative abundance $26.9 \%$ ) and in control women's endometrial and vaginal samples (mean relative abundance $49.9 \%$ and $42.9 \%$, respectively). On the other hand, $C$. parapsilosis was only detected in RPL women, with an average relative abundance of $18.2 \%$ in endometrial and $11.8 \%$ in vaginal samples.

\section{Comparison of microbiota in vaginal and endometrial samples}

To study the relationship between microbiota colonizing endometrium and vagina, we compared samples collected from these anatomical sites of the same woman. We found a strong within-individual correlation between vaginal and endometrial microbiota composition (mean Pearson's correlation coefficient $0.85, P<0.001$, Figure 4, Figure S5). The overlap was strongest in Lactobacillus-dominated samples, while intestinal and uncultured bacteria were more abundant in the endometrium, especially in RPL women.

\section{Data availability}

The sequencing data that support the findings of this study are available in the European Nucleotide Archive (PRJEB48310). All code has been deposited to GitHub: https://github.com/SchahzadSaqib/TOIVE. The clinical metadata are not publicly available due to privacy but are available from the corresponding author upon reasonable request. 


\section{Discussion}

We observed an association between RPL, reduced L. crispatus and increased $G$. vaginalis abundance in the endometrium, and an increased $G$. vaginalis abundance in the vagina. The composition of vaginal microbiota was in concordance with the endometrial microbiota.

Our finding of a reduced abundance of L. crispatus in RPL endometrial samples is in line with prior studies reporting that in vagina, $L$. crispatus dominance is considered a healthy microbial environment (Kindinger et al., 2017; Petrova et al., 2017). Furthermore, L. crispatus has been shown to be less abundant in the endometrium of patients with chronic endometritis (Liu et al., 2019), a condition associated with RPL (McQueen et al., 2021). In contrast, L. iners, which was the most dominant microbe in the RPL women's endometrium, has been associated with dysbiosis (Petrova et al., 2017) and adverse reproductive outcomes, including subfertility (Campisciano et al., 2021), spontaneous miscarriage (Nasioudis et al., 2017), and preterm birth (Kindinger et al., 2017). Our observations support the beneficial effect of $L$. crispatus and an unfavourable effect of $L$. iners on reproductive outcomes.

We also showed an association between endometrial and vaginal $G$. vaginalis colonization and RPL. $G$. vaginalis is typically dominant in BV, which has been associated with early (Haahr et al., 2019; Ralph et al., 1999) and especially with late miscarriages (Leitich and Kiss, 2007). Miscarriage has also been linked with Lactobacillus depletion and high bacterial diversity in vaginal samples collected during early pregnancy (Al-Memar et al., 2020). Kuon et al. (2017) reported that RPL women, who had vaginal G. vaginalis colonization, showed higher peripheral blood natural killer (NK) cell levels suggesting a link between dysbiotic reproductive tract microbiota, inflammation, and miscarriage. Furthermore, Lactobacillus depletion and the presence of pathogenic bacteria such as Bifidobacterium, Gardnerella, Klebsiella, and Neisseria in endometrial biopsies has been associated with unsuccessful reproductive outcomes in IVF treatment (Moreno et al., 2021).

Beneficial endometrial microbiota may support early pregnancy, while abnormal microbiota can be detrimental, although the underlying mechanisms are still poorly understood (Al-Nasiry et al., 2020; Bardos et al., 2020; Benner et al., 2018). In vitro, L. crispatus has been shown to attach to the decidualized endometrial cells and occupy the attachment sites from pathogenetic microbes (Shiroda and Manning, 2020). Conversely, unfavourable endometrial microbiota may weaken the epithelial tight junctions, allowing pathogens to enter the endometrial stroma and induce a harmful immune reaction (AlNasiry et al., 2020). The activation of Toll-like receptors on the surface of endometrial cells by microbial molecules may elicit the secretion of cytokines that alter the local immune environment (Benner et al., 2018), leading to poor NK cell maturation. This may provoke disturbances in placentation (Al-Nasiry et al., 
2020) since NK cells are essential in trophoblast invasion (Moffett and Shreeve, 2015) and remodelling of spiral arteries (Smith et al., 2009). Abnormal endometrial microbiota may also favour endometrial Thelper 1 cell types (Bardos et al., 2020), which is thought to predispose to RPL (Wang et al., 2020).

The origin of endometrial microbes is still unclear, but vagina and gastrointestinal tract have been suggested (Bardos et al., 2020). Our and others' (Walther-António et al., 2016; Wang et al., 2021) findings of the concordance between vaginal and endometrial microbiota speak in favour of the vaginal route. Other possible mechanisms include hematogenous spreading from the gastrointestinal tract or retrograde ascension from the peritoneal cavity through fallopian tubes. Interestingly, we detected oxygen-sensitive intestinal bacteria, including Blautia and Faecalibacterium spp., in endometrial samples. Also, Verstraelen et al. (2016) detected Bacteroides spp. in the endometrial samples of women with RPL or recurrent implantation failure which support the theory of peritoneal route. However, although we detected intestinal bacteria solely in the endometrial samples, it cannot be excluded that these bacteria have ascended from the perineum through vagina and remained under detection level due to unfavourable conditions, such as low pH. Finally, the role of intestinal vs. vaginal microbes in the uterus and their potential effects on reproductive health remain to be elucidated.

\section{Strengths and limitations}

To our knowledge, this is the first study comparing RPL women's endometrial microbiota with healthy controls. Our study population was ethnically and even genetically homogenous. Sampling was timed to the mid-secretory phase to analyse the microbiota during the receptive state of the endometrium, since the composition of vaginal (Lopes dos Santos Santiago et al., 2012) and endometrial microbiota (Kadogami et al., 2020; Khan et al., 2016) may vary throughout the menstrual cycle. We used a recommended technique, transcervical biopsy, for collecting endometrial tissue (Molina et al., 2021) because biopsies represent mucosal microbiota better than superficial samples, such as swabs or endometrial fluid (Liu et al., 2018). In addition to the microbiota, we also analysed the endometrial mycobiota, which has not been previously explored. Our control women represent the general population as closely as possible since they were healthy and did not have previous miscarriages or conditions known to associate with alterations in reproductive tract microbiota, such as endometriosis (Khan et al., 2016; Wei et al.,2020), endometrial polyps (Fang et al., 2016), PCOS (Tu et al., 2020), or fallopian tube occlusion (Haahr et al., 2019). We also studied the influence of various lifestyle factors on our findings. However, the applicability of our results to the general population remains to be explored.

As a limitation, low microbial abundance specimens, such as the endometrium, are susceptible to contamination (O'Callaghan et al., 2020). Although we avoided contact with the vagina during sampling, 
cervicovaginal contamination cannot be ruled out since similarities existed between individual vaginal and endometrial samples. However, growing evidence supports the theory that vaginal and endometrial ecosystems are not separate, but can share microbes (Chen et al., 2017; Walther-António et al., 2016; Wang et al., 2021), and only a minority of studies have questioned the existence of a uterine microbiota (Winters et al., 2019). Although cervical mucus protects the uterine environment, sperm pass from the vagina to the uterus and vaginally administered radioactively labelled albumin macrospheres spread in the uterine cavity within minutes (Kunz et al., 1997). Therefore, it is likely that microbes ascending from the vagina may colonize the endometrium. Also, the composition of endometrial microbiota has been reported to be highly similar in laparoscopically and trans-cervically taken samples (Chen et al., 2017), and common vaginal microbes, including lactobacilli, have been found in the endometrium even after hysterectomy (Chen et al., 2017; Miles et al., 2017; Mitchell et al., 2015). Overall, even if there were cervicovaginal contamination of our endometrial samples, it does not explain the observed differences between RPL women and controls.

Environmental contamination was not likely since bacteria found in the negative controls, such as $S$. oralis, were not found in women's samples, while reads for Lactobacillus and Gardnerella species were not substantial. The presence of vaginal microbes such as $F$. vaginae, $P$. amnii, and $S$. vaginae could be explained as contamination, however, considering their low abundance and prevalence in the study samples, as well as lack of significance within the group comparisons indicates that they had not significant effect on the overall results.

\section{Conclusions}

RPL was associated with an unfavourable female reproductive tract microbiota, especially in the uterus. An unhealthy endometrial microbial environment may be a new background cause for RPL, possibly contributing to an adverse immunological response during implantation and placentation. Further research should examine the mechanisms of how altered microbiota may contribute to RPL, evaluate if the prognosis of following pregnancies can be assessed according to the microbiota profile, and whether endometrial microbiota could be modified to increase the success of future pregnancies in some couples having RPL.

\section{Declarations}

\section{Acknowledgements}

We are deeply thankful for the women who participated in this study. We also wish to thank midwives Anneli Porkka, Sanna Erkkilä, and Karoliina Hyttinen for their excellent work in caring for the women and the schedules, laboratory coordinator Tinja Kanerva for processing the samples, and MD, MSc (Tech) Seppo Virtanen for his contribution to the study design. 
The European Union's Horizon 2020 research and innovation programme (H2020 MSCA Sweet Crosstalk project under grant agreement No. 814102) and the Department of Obstetrics and Gynaecology, Helsinki University Hospital (No. TYH2018232) funded the study. Funds from HUS Hyvinkää Hospital (No. M3080TUT21) and The Finnish Cultural Foundation for PP, Academy of Finland for IK and The Juhani Aho Foundation for Medical Research for PP and $\mathrm{HH}$.

\section{References}

Al-Memar, M., Bobdiwala, S., Fourie, H., Mannino, R., Lee, Y.S., Smith, A., Marchesi, J.R., Timmerman, D., Bourne, T., Bennett, P.R., Maclntyre, D.A., 2020. The association between vaginal bacterial composition and miscarriage: a nested case-control study. BJOG 127, 264-74

Al-Nasiry, S., Ambrosino, E., Schlaepfer, M., Morré, S.A., Wieten, L., Voncken, J.W., Spinelli, M., Mueller, M., Kramer, B.W., 2020. The Interplay Between Reproductive Tract Microbiota and Immunological System in Human Reproduction. Front. Immunol. 11, 378

Bardos, J., Fiorentino, D., Longman, R.E., Paidas, M., 2020. Immunological Role of the Maternal Uterine Microbiome in Pregnancy: Pregnancies Pathologies and Alterated Microbiota. Front. Immunol. 10, 2823

Bender Atik, R., Christiansen, O.B., Elson, J., Kolte, A.M., Lewis, S., Middeldorp, S., Nelen, W., Peramo, B., Quenby, S., Vermeulen, N., Goddijn, M., 2018. ESHRE guideline: recurrent pregnancy loss. Hum. Reprod. Open 2018:hoy004

Benner, M., Ferwerda, G., Joosten, I., van der Molen, R.G., 2018. How uterine microbiota might be responsible for a receptive, fertile endometrium. Hum. Reprod. Update 24, 393-415

Bradford, L.L., Ravel, J., 2017. The vaginal mycobiome: A contemporary perspective on fungi in women's health and diseases. Virulence. 8, 342-51

Campisciano, G., lebba, V., Zito, G., Luppi, S., Martinelli, M., Fischer, L., de Seta, F., Basile, G., Ricci, G., Comar, M., 2021. Lactobacillus iners and gasseri, prevotella bivia and hpv belong to the microbiological 
signature negatively affecting human reproduction. Microorganisms 9, 10.3390

Chen, C., Song, X., Wei, W., Zhong, H., Dai, J., Lan, Z., Li, F., Yu, X., Feng, Q., Wang, Z., Xie, H., Chen, X., Zeng, C., Wen, B., Zeng, L., Du, H., Tang, H., Xu, C., Xia, Y., Xia, H., Yang, H., Wang, Jian, Wang, Jun, Madsen, L., Brix, S., Kristiansen, K., Xu, X., Li, J., Wu, R., Jia, H., 2017. The microbiota continuum along the female reproductive tract and its relation to uterine-related diseases. Nat. Commun. 8, 875

Fang, R.L., Chen, L.X., Shu, W.S., Yao, S.Z., Wang, S.W., Chen, Y.Q., 2016. Barcoded sequencing reveals diverse intrauterine microbiomes in patients suffering with endometrial polyps. Am. J. Transl. Res. 8, 1581-92.

Freitas, A.C., Chaban, B., Bocking, A., Rocco, M., Yang, S., Hill, J.E., Money, D.M., Hemmingsen, S., Reid, G., Dumonceaux, T., Gloor, G., Links, M., O’Doherty, K., Tang, P., van Schalkwyk, J., Yudin, M., 2017. The vaginal microbiome of pregnant women is less rich and diverse, with lower prevalence of Mollicutes, compared to non-pregnant women. Sci. Rep. 7:9212-9

Giakoumelou, S., Wheelhouse, N., Cuschieri, K., Entrican, G., Howie, S.E.M., Horne, A.W., 2016. The role of infection in miscarriage. Hum. Reprod. Update 22, 116-33

Haahr, T., Zacho, J., Bräuner, M., Shathmigha, K., Skov Jensen, J., Humaidan, P., 2019. Reproductive outcome of patients undergoing in vitro fertilisation treatment and diagnosed with bacterial vaginosis or abnormal vaginal microbiota: a systematic PRISMA review and meta-analysis. BJOG 126, 200-7.

Kadogami, D., Nakaoka, Y., Morimoto, Y., 2020. Use of a vaginal probiotic suppository and antibiotics to influence the composition of the endometrial microbiota. Reprod. Biol. 20, 307-14

Khan, K.N., Fujishita, A., Masumoto, H., Muto, H., Kitajima, M., Masuzaki, H., Kitawaki, J., 2016. Molecular detection of intrauterine microbial colonization in women with endometriosis. Eur. J. Obstet. Gynecol. Reprod Biol. 199, 69-75 
Kindinger, L.M., Bennett, P.R., Lee, Y.S., Marchesi, J.R., Smith, A., Cacciatore, S., Holmes, E., Nicholson, J.K., Teoh, T.G., Maclntyre, D.A., 2017. The interaction between vaginal microbiota, cervical length, and vaginal progesterone treatment for preterm birth risk. Microbiome 5, 6-9

Koedooder, R., Mackens, S., Budding, A., Fares, D., Blockeel, C., Laven, J., Schoenmakers, S., 2019. Identification and evaluation of the microbiome in the female and male reproductive tracts. Hum. Reprod. Update 25, 298-325.

Korpela, K., 2016. Mare: Microbiota Analysis in R Easily. R package version 1.0 [WWW Document]. https://github.com/katrikorpela/mare.

Kunz, G., Beil, D., Deiniger, H., Einspanier, A., Mall, G., Leyendecker, G., 1997. The uterine peristaltic pump: Normal and impeded sperm transport within the female genital tract. Adv. Exp. Med. Biol. 424, 267-77.

Kuon, R.J., Togawa, R., Vomstein, K., Weber, M., Goeggl, T., Strowitzki, T., Markert, U.R., Zimmermann, S., Daniel, V., Dalpke, A.H., Toth, B., 2017. Higher prevalence of colonization with Gardnerella vaginalis and gram-negative anaerobes in patients with recurrent miscarriage and elevated peripheral natural killer cells. J. Reprod. Immunol. 120, 15-9

Leitich, H., Kiss, H., 2007. Asymptomatic bacterial vaginosis and intermediate flora as risk factors for adverse pregnancy outcome. Best Pract. Res. Clin. Obstet. Gynaecol. 21, 375-90

Liu, Y., Ko, E.Y.L., Wong, K.K.W., Chen, X., Cheung, W.C., Law, T.S.M., Chung, J.P.W., Tsui, S.K.W., Li, T.C., Chim, S.S.C., 2019. Endometrial microbiota in infertile women with and without chronic endometritis as diagnosed using a quantitative and reference range-based method. Fertil. Steril. 112, 707-17

Liu, Y., Wong, K.K.W., Ko, E.Y.L., Chen, X., Huang, J., Tsui, S.K.W., Li, T.C., Chim, S.S.C., 2018. Systematic comparison of bacterial colonization of endometrial tissue and fluid samples in recurrent miscarriage patients: Implications for future endometrial microbiome studies. Clin. Chem. 64, 1743-52 
Lopes dos Santos Santiago, G., Tency, I., Verstraelen, H., Verhelst, R., Trog, M., Temmerman, M., Vancoillie, L., Decat, E., Cools, P., Vaneechoutte, M., 2012. Longitudinal qPCR Study of the Dynamics of L. crispatus, L. iners, A. vaginae, (Sialidase Positive) G. vaginalis, and P. bivia in the Vagina. PLoS 0NE 7:e45281

Maclntyre, D.A., Chandiramani, M., Lee, Y.S., Kindinger, L., Smith, A., Angelopoulos, N., Lehne, B., Arulkumaran, S., Brown, R., Teoh, T.G., Holmes, E., Nicoholson, J.K., Marchesi, J.R., Bennett, P.R., 2015. The vaginal microbiome during pregnancy and the postpartum period in a European population. Sci. Rep. 5, 8988.

McQueen, D.B., Maniar, K.P., Hutchinson, A., Confino, R., Bernardi, L., Pavone, M.E., 2021. Redefining chronic endometritis: the importance of endometrial stromal changes. Fertil. Steril. 116, 855-61

McQueen, D.B., Perfetto, C.O., Hazard, F.K., Lathi, R.B., 2015. Pregnancy outcomes in women with chronic endometritis and recurrent pregnancy loss. Fertil. Steril. 104, 927-31

Miles, S.M., Hardy, B.L., Merrell, D.S., 2017. Investigation of the microbiota of the reproductive tract in women undergoing a total hysterectomy and bilateral salpingo-oopherectomy. Fertil. Steril. 107, 813-820.

Mitchell, C.M., Haick, A., Nkwopara, E., Garcia, R., Rendi, M., Agnew, K., Fredricks, D.N., Eschenbach, D., 2015. Colonization of the upper genital tract by vaginal bacterial species in nonpregnant women. Am. J. Obstet. Gynecol. 212, 611.e1-611.e9.

Moffett, A., Shreeve, N., 2015. First do no harm: Uterine natural killer (NK) cells in assisted reproduction. Hum. Reprod. 30, 1519-25

Molina, N.M., Sola-Leyva, A., Haahr, T., Aghajanova, L., Laudanski, P., Castilla, J.A., Altmäe, S., 2021. Analysing endometrial microbiome: Methodological considerations and recommendations for good practice. Hum. Reprod. 36, 859-79 
Moreno, I., Codoñer, F.M., Vilella, F., Valbuena, D., Martinez-Blanch, J.F., Jimenez-Almazán, J., Alonso, R., Alamá, P., Remohí, J., Pellicer, A., Ramon, D., Simon, C., 2016. Evidence that the endometrial microbiota has an effect on implantation success or failure. Am. J. Obstet. Gynecol. 215, 684-703.

Moreno, I., Garcia-Grau, I., Perez-Villaroya, D., Gonzalez-Monfort, M., Bahçeci, M., Barrionuevo, M.J., Taguchi, S., Puente, E., Dimattina, M., Lim, M.W., Meneghini, G., Aubuchon, M., Leondires, M., Izquierdo, A., Perez-Olgiati, M., Chavez, A., Seethram, K., Bau, D., Gomez, C., Valbuena, D., Vilella, F., Simon, C., 2021. Endometrial microbiota composition is associated with reproductive outcome in infertile patients. Microbiome. 2022; 10: 1

Nasioudis, D., Forney, L.J., Schneider, G.M., Gliniewicz, K., France, M., Boester, A., Sawai, M., Scholl, J., Witkin, S.S., 2017. Influence of Pregnancy History on the Vaginal Microbiome of Pregnant Women in their First Trimester. Sci. Rep. 7, 10201.

Oberle, A., Urban, L., Falch-Leis, S., Ennemoser, C., Nagai, Y., Ashikawa, K., Ulm, P.A., Hengstschläger, M., Feichtinger, M., 2021. 16S rRNA long-read nanopore sequencing is feasible and reliable for endometrial microbiome analysis. Reprod. Biomed. Online. 42, 1097-107.

O’Callaghan, J.L., Turner, R., Dekker Nitert, M., Barrett, H.L., Clifton, V., Pelzer, E.S., 2020. Re-assessing microbiomes in the low-biomass reproductive niche. BJOG 127, 147-158

Oksanen, J., Blanchet, F.G., Friendly, M., Kindt, R., Legendre, P., McGlinn, D., Minchin, P.R., O'Hara, R.B., Simpson, G.L., Solymos, P., Stevens, M.H.H., Szoecs, E., Wagner, H., 2020. Vegan: Community Ecology Package. R package version 2.5-7 [WWW Document]. https://CRAN.R-project.org/package=vegan.

Petrova, M.I., Reid, G., Vaneechoutte, M., Lebeer, S., 2017. Lactobacillus iners: Friend or Foe? Trends Microbiol. 25, 182-91.

Rai, R., Regan, L., 2006. Recurrent miscarriage. Lancet 368, 601-611 
Ralph, S.G., Rutherford, A.J., Wilson, J.D., 1999. Influence of bacterial vaginosis on conception and miscarriage in the first trimester: Cohort study. BMJ 319, 220-3

Saqib, S., 2021. Taxonomic annotations - BLAST alignment and text-mining based filtration in R [WWW Document]. https://github.com/SchahzadSaqib/taxminer.

Shiroda, M., Manning, S.D., 2020. Lactobacillus strains vary in their ability to interact with human endometrial stromal cells. PLoS One 15, e0238993

Smith, S.D., Dunk, C.E., Aplin, J.D., Harris, L.K., Jones, R.L., 2009. Evidence for immune cell involvement in decidual spiral arteriole remodeling in early human pregnancy. Am. J. Pathol. 174, 1959-71

Tu, Y., Zheng, G., Ding, G., Wu, Y., Xi, J., Ge, Y., Gu, H., Wang, Y., Sheng, J., Liu, X., Jin, L., Huang, H., 2020. Comparative Analysis of Lower Genital Tract Microbiome Between PCOS and Healthy Women. Front. Physiol. 11, 1108

Verstraelen, H., Vilchez-Vargas, R., Desimpel, F., Jauregui, R., Vankeirsbilck, N., Weyers, S., Verhelst, R., Sutter, P. de, Pieper, D.H., de Wiele, T. van, 2016. Characterisation of the human uterine microbiome in nonpregnant women through deep sequencing of the V1-2 region of the $16 \mathrm{~S}$ rRNA gene. PeerJ 4, e1602

Virtanen, S., Rantsi, T., Virtanen, A., Kervinen, K., Nieminen, P., Kalliala, I., Salonen, A., 2019. Vaginal Microbiota Composition Correlates Between Pap Smear Microscopy and Next Generation Sequencing and Associates to Socioeconomic Status. Sci. Rep. 9, 7750-8

Virtanen, S., Saqib, S., Kanerva, T., Nieminen, P., Kalliala, I., Salonen, A., 2021. Metagenome-validated Parallel Amplicon Sequencing and Text Mining-based Annotations for Simultaneous Profiling of Bacteria and Fungi: Vaginal Microbiota and Mycobiota in Healthy Women. https://www.researchsquare.com/article/rs-321778/v1 
Walther-António, M.R.S., Chen, J., Multinu, F., Hokenstad, A., Distad, T.J., Cheek, E.H., Keeney, G.L., Creedon, D.J., Nelson, H., Mariani, A., Chia, N., 2016. Potential contribution of the uterine microbiome in the development of endometrial cancer. Genome Med. 8, 122.

Wang, J., Li, Z., Ma, X., Du, L., Jia, Z., Cui, X., Yu, L., Yang, J., Xiao, L., Zhang, B., Fan, H., Zhao, F., 2021. Translocation of vaginal microbiota is involved in impairment and protection of uterine health. Nat. Commun. 12, 4191-8

Wang, W., Sung, N., Gilman-Sachs, A., Kwak-Kim, J., 2020. T Helper (Th) Cell Profiles in Pregnancy and Recurrent Pregnancy Losses: Th1/Th2/Th9/Th17/Th22/Tfh Cells. Front. Immunol .11, 2025

Wei, W., Zhang, X., Tang, H., Zeng, L., Wu, R., 2020. Microbiota composition and distribution along the female reproductive tract of women with endometriosis. Ann. Clin. Microbiol. Antimicrob. 19, 15

Winters, A.D., Romero, R., Gervasi, M.T., Gomez-Lopez, N., Tran, M.R., Garcia-Flores, V., Pacora, P., Jung, E., Hassan, S.S., Hsu, C.D., Theis, K.R., 2019. Does the endometrial cavity have a molecular microbial signature? Sci. Rep. 9, 9905

\section{Figures}

\section{Figure 1}

Flow chart describing the study design. RPL means recurrent pregnancy loss.

\section{Figure 2}

Stacked bar plots showing bacterial relative abundances in women with recurrent pregnancy loss (RPL) and control women, ordered based on top three most prevalent and abundant taxa (Lactobacillus crispatus, L. iners, and Gardnerella vaginalis). (A) Endometrial samples and (B) Vaginal samples. 
A)

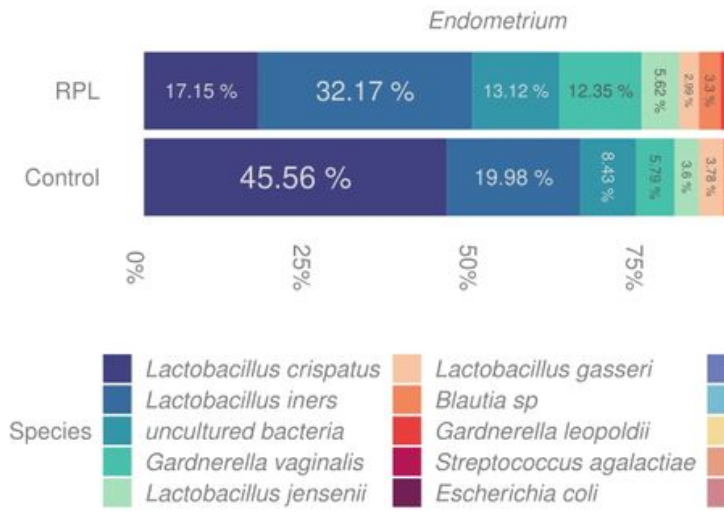

C)

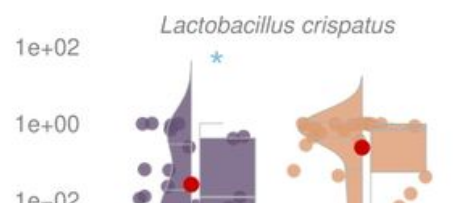

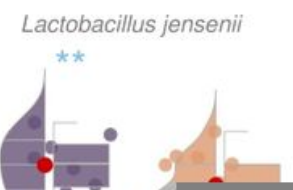

B)

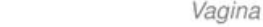

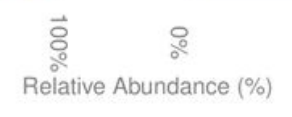

Lactobacillus sp Bifidobacterium breve

Faecalibacterium prausnitzi

Alloscardovia omnicolens

Fannyhessea vaginae

\section{$35.09 \% \quad 30.18 \%$}

$47.45 \%$

กั

जั जั

Bifidobacterium pseudocatenulatum Collinsella aerofaciens

Dialister invisus

Methylobacterium sp

Blautia faecis
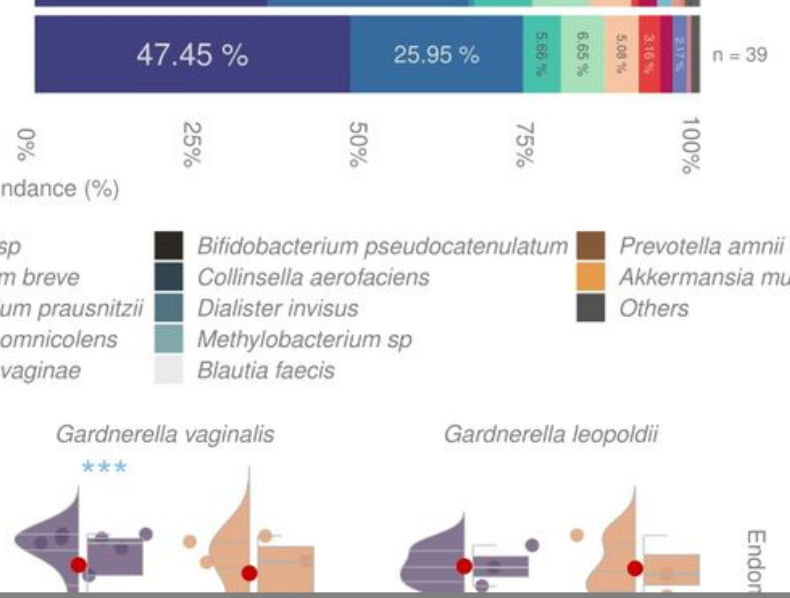

Gardnerella leopoldii

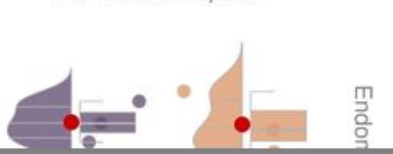

\section{Figure 3}

Illustration and summary of the main results and statistical analysis. (A-B) Bacterial mean relative abundances within the endometrial/vaginal and recurrent pregnancy loss (RPL)/control subgroups. (C -

E) Violin-Boxplots showing the distribution of data in each subgroup for taxa that were significantly differentially abundant after adjusting with age, parity, and BMI in the endometrial (C), vaginal (D) and pooled samples $(\mathrm{E})$. Asterisks indicate whether there are any statistically significant differences between the RPL and control (reference) subgroups; $* \star * q<0.001$, ${ }^{\star *} q<0.01,{ }^{*} q<0.05$. Pooled means the combined results of endometrial and vaginal samples. 
Figure 4

(A) Polar stacked bar plot illustrating the similarity of taxonomic profiles between endometrial (right) and vagina samples (left). The samples are arranged in the same order from top to bottom, creating a mirrorimage between the two semi-circles and allowing direct comparison between the sample types. (B) Pearson's correlation between the paired endometrial and vaginal samples, arranged in the same order.

\section{Supplementary Files}

This is a list of supplementary files associated with this preprint. Click to download.

- ToiveTableS1.xlsx

- ToiveTableS2.xlsx

- ToiveTableS3.xlsx

- ToiveFigures1.pdf

- ToiveFigures2.pdf

- ToiveFigureS3.pdf

- ToiveFigures4.pdf

- ToiveFigures5.pdf 